Check for updates

Cite this: RSC Adv., 2017, 7, 33837

Received 18th April 2017

Accepted 29th June 2017

DOI: $10.1039 / \mathrm{c} 7 \mathrm{ra04364c}$

rsc.li/rsc-advances

\section{Simple synthesis of SiGeaC porous microparticles as high-rate anode materials for lithium-ion batteries}

\author{
Yaguang Zhang, (D) Ning Du, ${ }^{*}$ Chengmao Xiao, Shali Wu, Yifan Chen, (D) Yangfan Lin, \\ Jinwei Jiang, Yuanhong He and Deren Yang*
}

\begin{abstract}
We report the synthesis of SiGeaC porous microparticles (PoSiGeaC) via the decomposition of $\mathrm{Mg}_{2} \mathrm{Si}$ / $\mathrm{Mg}_{2} \mathrm{Ge}$ composites, acid pickling and subsequent carbon coating processes, respectively. The content of $\mathrm{Ge}$ can be tuned by the initial ratio of $\mathrm{Mg}_{2} \mathrm{Si}$ and $\mathrm{Mg}_{2} \mathrm{Ge}$ in the composite. The as-synthesized PoSiGe@C has been used as the anode material of lithium-ion batteries, which shows an enhanced cyclic and rate performance compared to bare Si, PoSiGe as well as PoSi@C porous microparticles. Briefly, the PoSiGeaC delivers a good cycling stability with $70 \%$ capacity retention after 400 cycles and only $0.075 \%$ capacity loss per cycle at the current density of $0.8 \mathrm{~A} \mathrm{~g}^{-1}$. Furthermore, super rate capability is also expressed by the PoSiGeaC. The unique porous structure, and synergistic effect of $\mathrm{Si}$ and $\mathrm{Ge}$, may lead to the inherent high lithium-ion diffusivity and electrical conductivity of Ge, and good volume alleviation, which results in the good electrochemical performance.
\end{abstract}

\section{Introduction}

Alternative anode materials of lithium-ion batteries (LIBs) with high energy density have been pursued to take the place of the traditional carbonaceous anodes, because of the fast-growing improvement in the fields of consumer electronics and electrical vehicles. ${ }^{1-4}$

The group IV elements such as silicon (Si) and germanium (Ge) show high theoretical capacity, and are both promising candidates for the next-generation LIB anodes. ${ }^{5-7}$ For example, $\mathrm{Si}$ is very attractive for its abundant reserves, low price, low working potential $(<0.5 \mathrm{~V})$ and especially the highest theoretical capacity (3579 $\mathrm{mA} \mathrm{h} \mathrm{g}{ }^{-1}$, corresponding to $\left.\mathrm{Li}_{15} \mathrm{Si}_{4}\right){ }^{8-11}$ However, the huge volume variation during the lithiation and delithiation process leads to a poor cycle life, which hinders its commercial application. ${ }^{12-15}$ Compared with $\mathrm{Si}$, Ge also exhibits high theoretical capacity (1384 $\mathrm{mA} \mathrm{h} \mathrm{g}{ }^{-1}$, corresponding to $\mathrm{Li}_{15} \mathrm{Ge}_{4}$ ). Moreover, it shows a higher lithium ion diffusivity (400 times higher than $\mathrm{Si}$ ) and better electrical conductivity (104 times higher than Si).$^{16-20}$ Similarly, Ge suffers a volume expansion as large as $270 \%$ during the lithium-ion insertion. ${ }^{21,22}$ Moreover, the high cost and low abundance of Ge hinder its commercialization. ${ }^{23,24}$ Therefore, it is significant to take the advantage of both $\mathrm{Si}$ and $\mathrm{Ge}$ anodes, such as large capacity, relatively high lithium ion diffusivity and electrical conductivity. ${ }^{25-30}$ Moreover,

State Key Lab of Silicon Materials, School of Materials Science and Engineering, Cyrus Tang Center for Sensor Materials and Applications, Zhejiang University, Hangzhou 310027, People's Republic of China.E-mail: dna1122@zju.edu.cn; mseyang@zju. edu.cn; Fax: +86-571-87952322; Tel: +86-571-87953190 the volume alleviation should be improved to enhance the performance of Si and Ge anodes.

Recently, tremendous efforts have been contributed to study the $\mathrm{Si} / \mathrm{Ge}$ heterogeneous structures, such as nanostructured $\mathrm{Si}_{(1-x)} \mathrm{Ge}_{x}$ for tunable thin film lithium-ion battery anodes, ${ }^{24}$ $\mathrm{Si}_{x} \mathrm{Ge}_{1-x}$ nanoparticles, ${ }^{31} \mathrm{Si}-\mathrm{Ge}$ core-shell nanowires and $\mathrm{Ge}-\mathrm{Si}$ core-shell nanowires. ${ }^{32}$ Although the above examples have expressed the good rate performance benefiting from the contribution of Ge, their cycling stability is still unsatisfied due to the dramatic volume changes. To address this problem, the hollow structures like Si/Ge double-layered nanotube arrays and crystalline $\mathrm{Si} / \mathrm{Ge}$ hollow particles have been introduced. ${ }^{33-35}$ Although their cycling stability has been developed, the involved synthetic processes such as chemical vapor deposition and electro-reduction are too expensive for large-scale application. Therefore, it is of great significance to realize a simple method to synthesize $\mathrm{Si} / \mathrm{Ge}$ composites with unique morphology, good volume alleviation as well as good electrochemical performance.

Herein, we report a simple method to synthesize the uniform SiGe@C porous microparticles with the modification of our previous paper. ${ }^{36}$ Such a design of the porous and core-shell structure can buffer the volume change and enhance the conductivity. Furthermore, the addition of Ge has obtained multiple highlights: (1) the addition of Ge can act as the buffer layer which helps to hold up the huge volume change of Si. (2) The combination of Ge to Si can obtain relatively high lithium ion diffusivity and electrical conductivity, which is beneficial for its good rate performance. (3) During the process of lithiation, Ge first reacts with lithium at $\sim 0.42 \mathrm{~V}$ while Si reacts with 
lithium at $\sim 0.15 \mathrm{~V}$, indicating that the volume expansion of $\mathrm{Si}$ and Ge will not happen at the same time, which helps to release the swelling stress and improve the structural integrity profiting from this synergistic effect. Therefore, the good cycling and rate performance of PoSiGe@C can be expected.

\section{Experimental}

\subsection{Synthesis of SiGe@C porous microparticles}

$\mathrm{Mg}_{2} \mathrm{Si}$ (purity, >99\%) and $\mathrm{Mg}_{2} \mathrm{Ge}$ (purity, >99\%) powder were purchased from Alfa Aesar. Bare SiGe porous microparticles (PoSiGe) were synthesized via the following procedures. Typically, $\mathrm{Mg}_{2} \mathrm{Si}, \mathrm{Mg}_{2} \mathrm{Ge}$ and grinding balls with a mass ratio of $1: 0.4: 2.6$ were ground in a planetary ball mill (QM-3SP2, Nanjing University Company) at $400 \mathrm{rpm}$ for $3 \mathrm{~h}$. After the ball-milling process, the mixture was then heated to $650{ }^{\circ} \mathrm{C}$ at a heating rate of $5{ }^{\circ} \mathrm{C} \mathrm{min}^{-1}$ under air atmosphere, keeping for $12 \mathrm{~h}$ in the furnace. After cooling down to room temperature, the powder was washed with hydrochloric acid ( $\mathrm{HCl})$ solution to remove $\mathrm{MgO}$. Final product was obtained after a centrifuging process using the deionized water and alcohol for three times, respectively. Thermal decomposition of acetylene gas was carried out at $600{ }^{\circ} \mathrm{C}$ for $3 \mathrm{~h}$ in a quartz furnace to deposit the carbon layer. In addition, the bare Si and Si@C porous structures were synthesized via the same procedure in absence of $\mathrm{Mg}_{2} \mathrm{Ge}$.

\subsection{Characterization of the products}

The crystal structures of the materials were measured by a high power X-ray diffraction (XRD) on a Rigaku D/max-ga X-ray diffractometer, where the $\mathrm{Cu} \mathrm{K}$ radiation was $1.54 \AA$. The morphology and structure of the materials were characterized by scanning electron microscopy (SEM, HITACH S4800) and transmission electron microscopy (TEM, PHILIPS F200). Energy dispersive spectroscopy (EDS) patterns were recorded by a Tecnai G2 F20 Chemi STEM attached with an Oxford X-Max 80T EDX detector system. Raman spectra were recorded on a HR800 Raman spectrometer using the $514 \mathrm{~nm}$ line of an Ar ion laser operated at $10 \mathrm{~mW}$. Thermogravimetric analysis (TGA) was conducted using a SDT Q600 V8.2 Build 100. BrunauerEmmett-Teller (BET) surface area and pore volume were recorded using a Beckman Coulter Omnisorp100cx.

\subsection{The electrochemical performance of the products}

To determine the electrochemical properties of the products, coin-type half cells composed of the active materials (SiGe, PoSiGe@C or PoSi@C) as the working electrode and the lithium metal as the counter-electrode were prepared in an Ar-filled glove box (Mbraun, labstar, Germany). The work electrode was made of the active materials, super $P$ carbon black, and sodium carboxymethyl cellulose (Aldrich) binder in a weight ratio of $70: 15: 15$. The electrolyte solution was composed of $1 \mathrm{M} \mathrm{LiPF}_{6}$ in ethylene carbonate/dimethyl carbonate (EC/DMC, 1:1 in volume). 5 vol\% fluoroethylene carbonate (FEC) was added in the electrolyte solution. Before the test, the cells were aged for $24 \mathrm{~h}$. Charge and discharge were cycled on a Land CT2001A system at the potentials between 0.01 and $1.5 \mathrm{~V}$. Cyclic voltammetry was also carried out on an Arbin BT 2000 system at a scan rate of $0.1 \mathrm{mV} \mathrm{s}^{-1}$. The electrochemistry impedance spectroscopy (EIS) was measured on a CHI660D electrochemistry workstation, the amplitude used to the cells was $5 \mathrm{mV}$ and the frequency range was from $0.01 \mathrm{~Hz}$ to $100 \mathrm{kHz}$.

\section{Results and discussion}

SiGe porous microparticles are achieved via the annealing of $\mathrm{Mg}_{2} \mathrm{Si} / \mathrm{Mg}_{2} \mathrm{Ge}$ composites, and subsequent acid treatment. Firstly, $\mathrm{Mg}_{2} \mathrm{Si}$ and $\mathrm{Mg}_{2} \mathrm{Ge}$ are not stable at high temperatures and can decompose to $\mathrm{Mg}$ and $\mathrm{M}(\mathrm{M}=\mathrm{Si}, \mathrm{Ge})$. Specially, $\mathrm{Mg}$ is very active and can be oxidized easily even under an atmosphere of low oxygen content. Meanwhile, the consumption of $\mathrm{Mg}$ can boost the further decomposition of $\mathrm{Mg}_{2} \mathrm{Si}$ and $\mathrm{Mg}_{2} \mathrm{Ge}$, until they completely convert to $\mathrm{M}(\mathrm{M}=\mathrm{Si}, \mathrm{Ge})$ and $\mathrm{MgO}$. Then, the PoSiGe can be obtained via the acid pickling to totally remove the by-product $(\mathrm{MgO})$. The X-ray diffraction (XRD) analysis has been employed to illustrate the above-mentioned process (Fig. 1a). As can be seen, the peaks of the initial $\mathrm{Mg}_{2} \mathrm{Si} / \mathrm{Mg}_{2} \mathrm{Ge}$ composites are consistent with cubic $\mathrm{Mg}_{2} \mathrm{Si}$ (JCPDS No. 01-0750445) and cubic $\mathrm{Mg}_{2} \mathrm{Ge}$ (JCPDS No. 02-1135). ${ }^{36}$ After the annealing process, the peaks of $\mathrm{Mg}_{2} \mathrm{Si}$ and $\mathrm{Mg}_{2} \mathrm{Ge}$ disappear, while the peaks of $\mathrm{MgO}, \mathrm{Si}$ and Ge emerge, indicating the complete transformation from $\mathrm{Mg}_{2} \mathrm{M}$ to $\mathrm{MgO}$ and $\mathrm{M}(\mathrm{M}=\mathrm{Ge}$, $\mathrm{Si}$ ). After the acid treatment, $\mathrm{MgO}$ is removed by $\mathrm{HCl}$ solution, at the same time, only $\mathrm{Si}$ and Ge peaks can be detected. It can be seen that the apparent diffraction peaks at $28^{\circ}, 47^{\circ}, 56^{\circ}$ are typical Si peaks which are assigned to its $\{111\},\{220\}$, and $\{311\}$ planes respectively. The rest diffraction peaks located at $27^{\circ}$, $45^{\circ}, 53^{\circ}$ are corresponding to the $\{111\},\{220\}$, and $\{311\}$ planes of crystalline cubic Ge. It should be mentioned that the peaks of crystalline cubic Ge are wider than standard peaks because of its the composed nano-sized particles, according to the Scherrer formula. According to the result of EDS in Fig. 1b, the weight ratio of $\mathrm{Si}$ to $\mathrm{Ge}$ is measured to $3: 2$, which is roughly agreed with the initial ratio of $\mathrm{Mg}_{2} \mathrm{Si}$ and $\mathrm{Mg}_{2} \mathrm{Ge}$.

The morphology of PoSiGe is further investigated through the scanning electron microscopy (SEM) and transmission electron microscopy (TEM) analysis. From the low magnification SEM image of Fig. 2a, all particles of the sample show porous structure with the size of $0.5-3 \mu \mathrm{m}$. And clear porous features are demonstrated by the magnified SEM image (Fig. 2b), in which the pores with a diameter of tens of nanometers distribute uniformly in the whole particle. The characteristic of porosity is also exhibited by the TEM image (Fig. 2c). Furthermore, the high-resolution TEM (HRTEM) image provides more details that go deep into the crystal lattice. As shown in Fig. 2e, the wide lattice fringes of $0.31 \mathrm{~nm}$ are wellmatched to the $\{111\}$ crystal planes of $\mathrm{Si}$, while the narrow lattices are measured to be $0.2 \mathrm{~nm}$ which are corresponding with the $\{220\}$ crystal planes of Ge. The element distribution is further studied by the energy-dispersive X-ray (EDX) underscanning TEM (STEM) mode. Elements mapping is measured in Fig. 2f, which shows a uniform distribution of Si and Ge. 

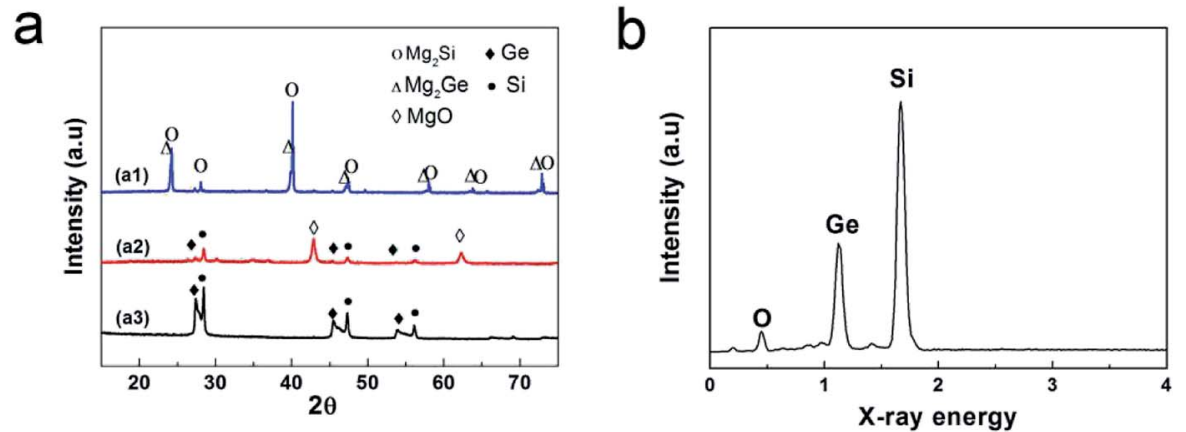

Fig. 1 (a) Evolution of the XRD pattern from the raw $\mathrm{Mg}_{2} M(M=S i, G e)$ materials to the PoSiGe. (b) EDS pattern of the as-synthesized PoSiGe.

The carbon layer has been further coated onto the surface of PoSiGe via the pyrolysis of acetylene. Fig. 3 shows the morphological and structural characterization of PoSiGe@C. It can be seen from Fig. 3a-b that the porous structures have been retained after the deposition of the carbon layer. However, the pores seem shrinking and the surface of the particles turns to smooth due to the deposition of carbon layer. Fig. 3c-d show the TEM and HRTEM images of an individual PoSiGe@C particle, which further confirms the porous structures. Moreover, the carbon layer with a thickness of $\sim 15 \mathrm{~nm}$ is identified by the HRTEM image.

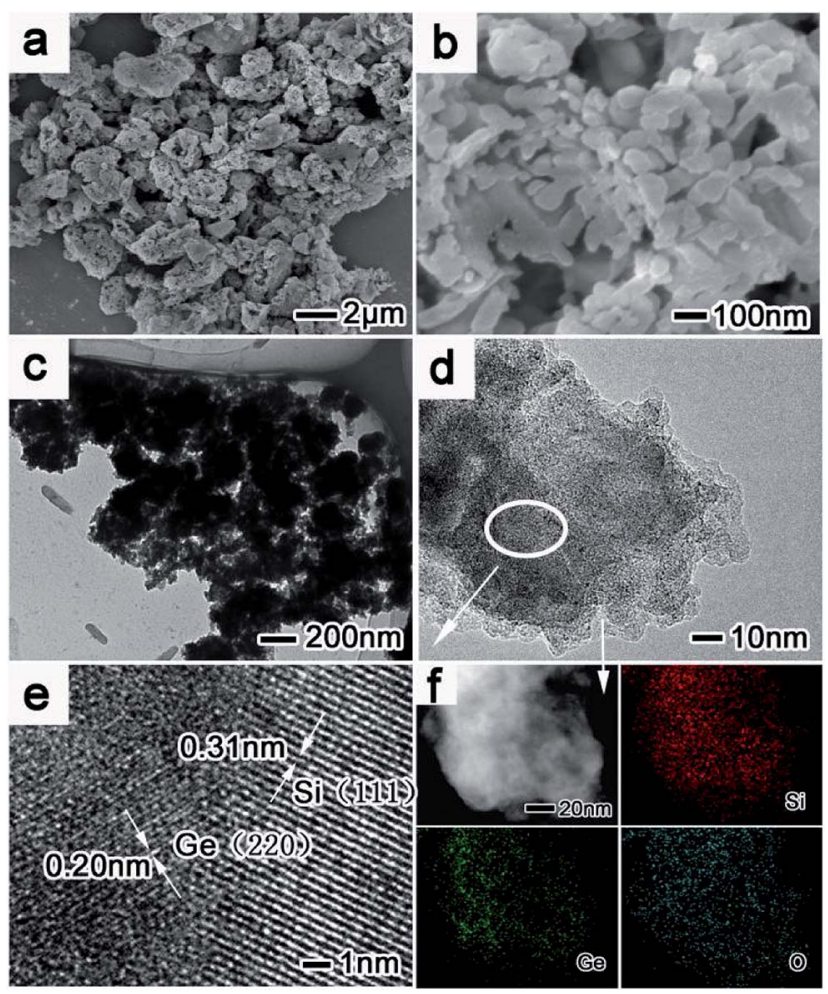

Fig. 2 The morphology of PoSiGe: (a and b) SEM images at different magnification showing the characteristic of porous structure; ( $c$ and d) TEM images at different magnification; (e) HRTEM image of the highlight area in (d); (e) EDX elemental maps showing signals from $\mathrm{Si}$ (Red), Ge (Green) and $O$ (Blue) respectively, indicating complete uniform distribution of $\mathrm{Si}$ and $\mathrm{Ge}$.
Fig. 4a shows the Raman spectrum of the as-synthesized PoSiGe@C. As observed, two characterization peaks located at $1340 \mathrm{~cm}^{-1}$ (D band) and $1580 \mathrm{~cm}^{-1}$ (G band) represent the amorphous and graphitizing features of the carbon layer, respectively. ${ }^{22}$ Another two peaks located at $293 \mathrm{~cm}^{-1}$ and 516 $\mathrm{cm}^{-1}$ correspond to the vibration of $\mathrm{Ge}-\mathrm{Ge}$ and $\mathrm{Si}-\mathrm{Si},{ }^{25}$ indicating the co-existing of Ge and Si components. It should be mentioned that no $\mathrm{Si}-\mathrm{Ge}$ peaks can be observed. The carbon content in the products is identified by thermal gravity analysis (TGA) in Fig. 4b. From the TGA curve, a fast mass fading is observed between $400{ }^{\circ} \mathrm{C}$ and $550{ }^{\circ} \mathrm{C}$, following with a stable zone at higher temperatures. The above mass loss is due to the oxidation of carbon into gaseous pieces. ${ }^{37}$ Accordingly, it is roughly estimated that the content of the carbon is $20.6 \mathrm{wt} \%$. Besides, it should be mentioned that the content of carbon can be adjusted by the reacting conditions such as the reacting temperature and time. Furthermore, XPS spectra have been employed in the Fig. $4 \mathrm{c}-\mathrm{d}$ to analyze the state of $\mathrm{Si}$ and Ge. It shows that Ge mainly exist in $0^{+}$vacancy and a small part of Ge

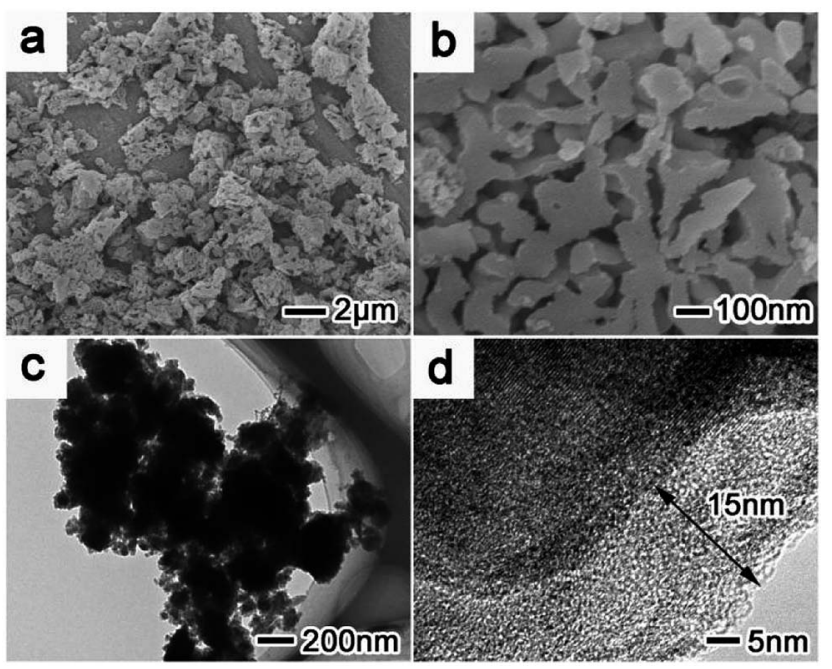

Fig. 3 The morphology of PoSiGeaC: ( $a$ and b) SEM images at different magnification, showing that the characteristic of porous structure is still maintained after carbon coating; (c) TEM image; (d) HRTEM image showing the amorphous carbon shell with thickness of $15 \mathrm{~nm}$. 

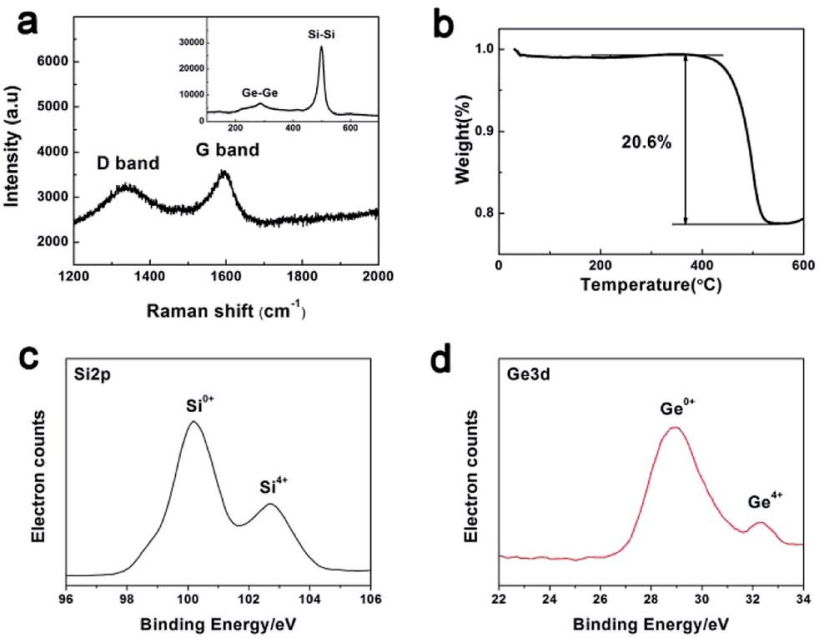

Fig. 4 Characterization of the PoSiGeaC: (a) Raman spectrum; (b) TGA analysis showing the content of carbon component; (c) Si2p spectra of XPS; (d) Ge3d spectra of XPS.

exist in $4^{+}$vacancy. Therefore, it can be proved that the final product contains a large amount of germanium rather than germanium dioxide. Similarly, Si mainly exists in $0^{+}$vacancy and a small part of Si exist in $4^{+}$vacancy.

To further study the porosity of the composite, Fig. 5ad show the isotherm curve from Brunauer-Emmett-Teller (BET) analysis and the pore size distribution of the PoSiGe and PoSiGe@C, respectively. It can be measured that the PoSiGe have a BET surface area of $70.35 \mathrm{~m}^{2} \mathrm{~g}^{-1}$ with an average $\mathrm{BJH}$ pore diameter of $\sim 40 \mathrm{~nm}$. After the carbon coating process, the PoSiGe@C shows a smaller BET surface area of $62.08 \mathrm{~m}^{2} \mathrm{~g}^{-1}$ and similar pore size distribution. It is the porous microstructures that can facilitate fast lithium-ion diffusion and accommodate the volume change during the lithiation and delithiation, resulting in excellent electrochemical performance.
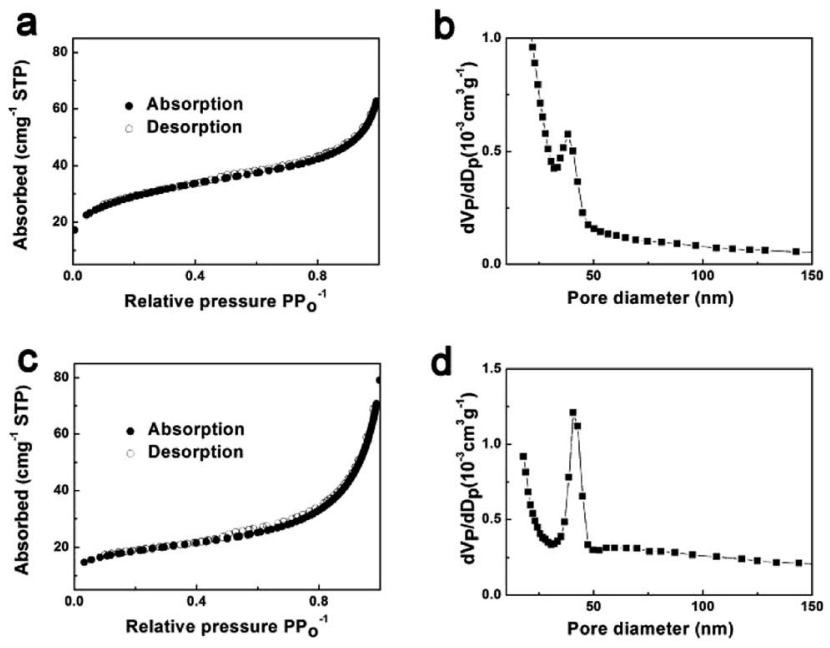

Fig. 5 ( $\mathrm{a}$ and $\mathrm{c}$ ) Nitrogen adsorption and desorption isotherms profile for PoSiGe and PoSiGeac, respectively; (b and d) the corresponding pore-size distribution curve for PoSiGe and PoSiGe@C, respectively.
As anodes of the lithium-ion batteries, the lithiation/ delithiation process of the PoSiGe@C electrodes are investigated by cyclic voltammetry (CV) curves which cycled between 0.01 and $1.5 \mathrm{~V}$ at a scanning rate of $0.1 \mathrm{mV} \mathrm{s}^{-1}$. As shown in the first discharge process (Fig. 6a), the cathodic peak located at $0.42 \mathrm{~V}$ is contributed to the Li-alloying reaction with Ge, and another cathodic peak at $0.14 \mathrm{~V}$ is attributed to the lithiation of $\mathrm{Si}^{24}$ In terms of the first charge process, there are two anodic peaks occurring at 0.34 and $0.55 \mathrm{~V}$ during the delithiation process for Ge electrode. ${ }^{7}$ And similarly two peaks at 0.33 and $0.49 \mathrm{~V}$ can be attributed to Si electrode. ${ }^{38}$ Therefore, the two peaks are supposed to the dealloying reactions of $\mathrm{Li}-\mathrm{Ge}$ as well as $\mathrm{Li}-\mathrm{Si}$. In addition, the cathodic peak at $\sim 0.42 \mathrm{~V}$ shifts to higher voltage during the next two cycles, which is related with change of Ge or Si from crystalline to amorphous after the first delithiation process..$^{25}$ It should be pointed out that no other change appears in the second and third cyclic voltammetry curves, suggesting the good cycling stability. The cycling performance of the bare Si, PoSiGe and PoSiGe@C are evaluated at $0.4 \mathrm{~A} \mathrm{~g}^{-1}$ between 0.01 and $1.5 \mathrm{~V}$ (Fig. 6b). It can be seen that the bare Si shows the rapid capacity fade, resulting in a capacity of $205 \mathrm{~mA} \mathrm{~h} \mathrm{~g}^{-1}$ after 15 cycles with only $10 \%$ capacity retention. For comparison, the PoSiGe exhibit better cycling performance than bare Si, which expresses a capacity of $403 \mathrm{~mA} \mathrm{~h} \mathrm{~g}{ }^{-1}$ after 15 cycles and achieve relatively high capacity retention of $20 \%$. The addition of Ge can improve the electric conductivity, which may be responsible for the improved cycling stability. However, the performance of PoSiGe is still unsatisfied because the porous structure can buffer the volume change to some extent, ${ }^{18,37}$ which can't prevent the further pulverization and capacity fading. Furthermore, the remarkable improved cycling stability is demonstrated by the PoSiGe@C electrode, which delivers a high reversible capacity of $978 \mathrm{~mA} \mathrm{~h} \mathrm{~g}{ }^{-1}$ after 15 cycles with $92.3 \%$ capacity retention. It is indicated that the carbon layer can protect the SiGe from side reaction with the electrolyte and
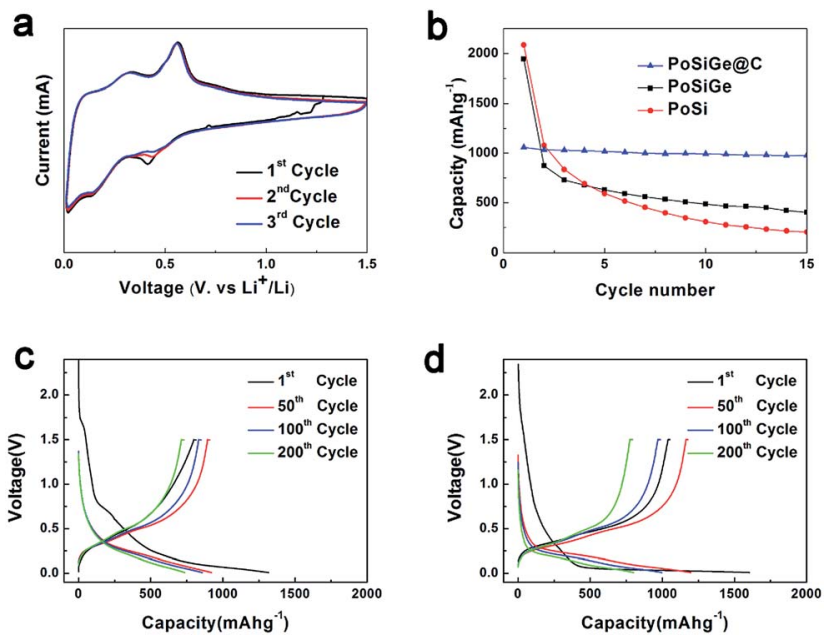

Fig. 6 (a) Current-voltage (CV) curves for the first three cycles of the PoSiGeaC; (b) the capacity versus cycle numbers of the bare $\mathrm{Si}$, PoSiGe and PoSiGeaC at $0.4 \mathrm{~A} \mathrm{~g}^{-1}$, respectively; (c and d) voltage profiles plotted of PoSiGeaC and PoSiaC for the 1st, 50th, 100th, 200th cycles at $0.8 \mathrm{~A} \mathrm{~g}^{-1}$. 
stabilize the structure during the alloying/de-alloying process, which facilitate the cycling performance.

To further compare the electrochemical characteristics of the PoSiGe@C and PoSi@C electrodes, voltage profiles at a current density of $0.8 \mathrm{~A} \mathrm{~g}^{-1}$ between 0.01 and $1.5 \mathrm{~V}$ are recorded in Fig. 6c-d. At the first discharge process, voltage plateau appears at $\sim 0.6 \mathrm{~V}$, which is owing to the reaction between $\mathrm{Li}$ and $\mathrm{GeO}_{2}$. The voltage plateau at $0.6 \mathrm{~V}$ is consistent with the charge-discharge profiles of $\mathrm{Ge} / \mathrm{GeO}_{2}$ anode in previous paper. ${ }^{39}$ At the following cycles, the discharge voltage plateau of PoSiGe@C turns into the sloping curves starting from $0.5 \mathrm{~V}$, and the sloping curves of PoSi@C starting from the lower voltage of $0.25 \mathrm{~V}$, because $\mathrm{Ge}$ reacts with $\mathrm{Li}$ at higher voltage than Si. Moreover, the capacity retention of PoSiGe@C electrode is better than PoSi@C, indicating the better cycling stability of PoSiGe@C.

Fig. 7a shows the long-term cycling performance for both PoSiGe@C and PoSi@C electrodes. As described, the PoSiGe@C shows the initial charge capacity of $800 \mathrm{~mA} \mathrm{~h} \mathrm{~g}^{-1}$ and decreases to $560 \mathrm{~mA} \mathrm{~h} \mathrm{~g}^{-1}$ after 400 cycles with $70 \%$ capacity retention. In comparison, the PoSi@C display an initial charge capacity of $1153 \mathrm{~mA} \mathrm{~h} \mathrm{~g}^{-1}$ and decrease to $500 \mathrm{~mA} \mathrm{~h} \mathrm{~g}^{-1}$ after 400 cycles with only $43 \%$ capacity retention. Although the PoSiGe@C exhibit lower initial capacity due to the relatively lower theoretical capacity of Ge, the capacity of the PoSiGe@C surpass the PoSi@C after 280 cycles, indicating the better cycling stability. Besides, the corresponding coulombic efficiency of PoSiGe@C quickly increase from $63 \%$ for the first cycle to $98 \%$ after 6 cycles, and further reach a stable value of $99.5 \%$ after several cycles. The high coulombic efficiency is very meaningful especially for the full-cells that contain limited lithium. What is more, the rate capability of both PoSiGe@C and PoSi@C electrodes is recorded in Fig. $7 \mathrm{~b}$. At the current density of $0.4,0.8$, $1.6,3.2$, and $6.4 \mathrm{Ag}^{-1}$, the PoSiGe@C achieve capacities of 1000 , $870,690,440$, and $420 \mathrm{~mA} \mathrm{~h} \mathrm{~g}^{-1}$, respectively. For comparison,
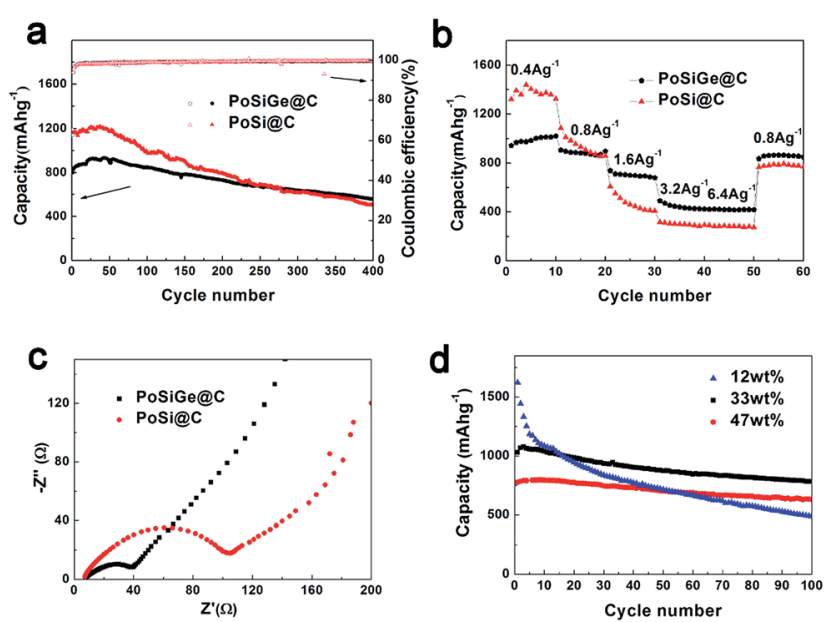

Fig. 7 (a) Long-term cycling performance for PoSiGe@C and PoSi@C at the current density of $0.8 \mathrm{~A} \mathrm{~g}^{-1}$; (b) rate capacity for PoSiGe@C and PoSiaC at different current densities; (c) Nyquist plots for PoSiGeaC and PoSiaC electrodes; (d) cycling performance of the PoSiGeaC with different Ge content at the current of $0.4 \mathrm{~A} \mathrm{~g}^{-1}$. the PoSi@C display capacities of 1380, 920, 450, 290, and 280 $\mathrm{mA} \mathrm{h} \mathrm{g}^{-1}$, respectively. It is obvious that the PoSiGe@C delivers better rate capability than PoSi@C. Especially, the capacity of PoSiGe@C is recovered to $860 \mathrm{~mA} \mathrm{~h} \mathrm{~g}{ }^{-1}$ when the current is returned to $0.8 \mathrm{~A} \mathrm{~g}^{-1}$, while the PoSi@C can only restore to 780

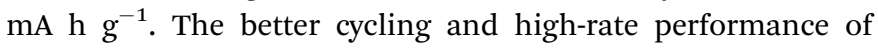
PoSiGe@C can be attributed to the integration of Ge that can give higher lithium-ion diffusivity and electrical conductivity.

In order to intuitively illustrate the advantage of $\mathrm{Ge}$, electrochemical impedance spectroscopy (EIS) was measured on the above-mentioned two electrodes. As displayed in Fig. 7c, all the spectra consist of a depressed semicircle in the high-to-medium frequency range and a straight line in the low frequency range. It is clearly that the diameter of the semicircle in the PoSiGe@C electrode is smaller than that of the PoSi@C electrode, indicating lower charge-transfer impedances. This is due to the addition of Ge that improves the electrical conductivity of the electrode. The effects of Ge on the electrochemical performance of PoSiGe@C electrode have been also investigated. Fig. 7d presents the discharge capacity $v s$. cycle number with the different Ge contents at a current density of $0.4 \mathrm{~A} \mathrm{~g}^{-1}$. It can be seen that, sample A with $47 \mathrm{wt} \%$ Ge shows an initial reversible capacity of $777 \mathrm{~mA} \mathrm{~h} \mathrm{~g}^{-1}$ and decreases to $632 \mathrm{~mA} \mathrm{~h} \mathrm{~g}^{-1}$ after 100 cycles, with the capacity retention of $81.3 \%$. In contrast, the initial reversible capacities of sample B (Ge, $33 \mathrm{wt} \%)$ and sample $\mathrm{C}(\mathrm{Ge}, 12 \mathrm{wt} \%)$ are 1030 and $1621 \mathrm{~mA} \mathrm{~h} \mathrm{~g}^{-1}$, which decreases to 783 and $487 \mathrm{~mA} \mathrm{~h} \mathrm{~g}^{-1}$ after 100 cycles, indicating the capacity retention of $76.0 \%$ and $30.0 \%$, respectively. It can be concluded that the sample with higher Ge contents can exhibit better cycling performance. This result can further confirm the role of Ge that can improve the lithium ion diffusivity and electrical conductivity.

\section{Conclusions}

In summary, PoSiGe@C was synthesized via the transformation of $\mathrm{Mg}_{2} \mathrm{Si} / \mathrm{Mg}_{2} \mathrm{Ge}$ composites, and the pyrolysis of acetylene. When used as anode materials of lithium-ion batteries, the assynthesized PoSiGe@C deliver a capacity of $560 \mathrm{~mA} \mathrm{~h} \mathrm{~g}^{-1}$ with $70 \%$ capacity retention after 400 cycles and only $0.075 \%$ capacity loss per cycle at the current density of $0.8 \mathrm{~A} \mathrm{~g}^{-1}$. Moreover, PoSiGe@C shows better cycling and rate performance compared to PoSi@C. The higher content of Ge can lead to better cycling performance. It is indicated that the addition of Ge can improve the lithium ion diffusivity and electrical conductivity, which result in the better cycling and rate performance.

\section{Conflict of interest}

There are no conflicts of interest to declare.

\section{Acknowledgements}

The authors would like to appreciate the financial supports from the Fundamental Research Funds for the Central Universities (2016FZA4007) and the Program for Innovative Research 
Team in University of Ministry of Education of China (IRT13R54).

\section{References}

1 J. M. Tarascon and M. Armand, Nature, 2001, 414, 359-367. 2 Y. Sun, N. Liu and Y. Cui, Nat. Energy, 2016, 1, 16071.

3 H. Gao, I. Plümel, L. Xiao, G. Xu, Y. Ren, X. Zuo, Y. Liu, C. Schulz, H. Wiggers, K. Amine and Z. Chen, Nano Lett., 2017, 17, 1512-1519.

4 V. Aravindan, Y. Lee and S. Madhavi, Adv. Energy Mater., 2015, 5, 140225.

5 T. Kennedy, M. Brandon and K. M. Ryan, Adv. Mater., 2016, 28, 5696-5704.

6 Z. Hu, S. Zhang, C. Zhang and G. Cui, Coord. Chem. Rev., 2016, 326, 34-85.

7 H. Jia, R. Kloepsch, X. He, J. P. Badillo, P. Gao, O. Fromm, T. Placke and M. Winter, Chem. Mater., 2014, 26, 5683-5688.

8 T. Kasukabe, H. Nishihara, K. Kimura, T. Matsumoto, H. Kobayashi, M. Okai and T. Kyotani, Sci. Rep., 2016, 7, 42734.

9 M. Ko, P. Oh, S. Chae, W. Cho and J. Cho, Small, 2015, 11, 4058-4073.

10 M. Gu, Y. He, J. Zheng and C. Wang, Nano Energy, 2015, 17, 366-383.

11 X. Zuo, Y. Xia, Q. Ji, X. Gao, S. Yin, M. Wang, X. Wang, B. Qiu, A. Wei, Z. Sun, Z. Liu, J. Zhu and Y. Cheng, ACS Nano, 2017, 11, 889-899.

12 C. Li, T. Shi, H. Yoshitake and H. Wang, Electrochim. Acta, 2017, 225, 11-18.

13 S. J. Lee, H. J. Kim, T. M. Hwang, S. Choi, S. H. Park, E. Deniz, D. S. Jung and J. W. Choi, Nano Lett., 2017, 17, 1870-1876.

14 A. Magasinski, P. Dixon, B. Hertzberg, A. Kvit, J. Ayala and G. Yushin, Nat. Mater., 2010, 9, 353-358.

15 M. L. Terranova, S. Orlanducci, E. Tamburri, V. Guglielmotti and M. Rossi, J. Power Sources, 2014, 246, 167-177.

16 C. Y. Chou and G. S. Hwang, J. Power Sources, 2014, 263, 252258.

17 T. D. Bogart, A. M. Chockla and B. A. Korgel, Curr. Opin. Chem. Eng., 2013, 2, 286-293.

18 G. H. Yue, X. Q. Zhang, Y. C. Zhao, Q. S. Xie, X. X. Zhang and D. L. Peng, RSC Adv., 2014, 4, 21450-21455.

19 Y. Domi, H. Usui, Y. Takemoto, K. Yamaguchi and H. Sakaguchi, Chem. Lett., 2016, 45, 1198-1200.

20 Z. Wang, Q. Su, H. Deng, W. He, J. Lin and Y. Q. Fu, J. Mater. Chem. A, 2014, 2, 13976-13982.
21 S. Li, C. Chen, K. Fu, L. Xue, C. Zhao, S. Zhang, Y. Hua, L. Zhou and X. Zhang, Solid State Ionics, 2014, 254, 17-26.

22 K. H. Seng, M. H. Park, Z. P. Guo, H. K. Liu and J. Cho, Angew. Chem., Int. Ed. Engl., 2012, 51, 5657-5661.

23 W. Luo, D. Shen, R. Zhang, B. Zhang, Y. Wang, S. X. Dou, H. K. Liu and J. Yang, Adv. Funct. Mater., 2016, 26, 78007806.

24 P. R. Abel, A. M. Chockla, Y. M. Lin, V. C. Holmberg, J. T. Harris, B. A. Korgel, A. Heller and C. B. Mullins, ACS Nano, 2013, 7, 2249-2257.

25 N. Lin, L. Wang, J. Zhou, J. Zhou, Y. Han, Y. Zhu, Y. Qian and C. Caob, J. Mater. Chem. A, 2015, 3, 11199-11202.

26 M. H. Park, Y. H. Cho, K. Kim, J. Kim, M. Liu and J. Cho, Angew. Chem., Int. Ed., 2011, 50, 9647-9650.

27 H. Kim, Y. Son, C. Park, M. J. Lee, M. Hong, J. Kim, M. Lee, J. Cho and H. C. Choi, Nano Lett., 2015, 15, 4135-4142.

28 J. Wang, N. Du, H. Zhang, J. Yu and D. Yang, J. Mater. Chem., 2011, 22, 1511-1515.

29 T. Kennedy, M. Bezuidenhout, K. Palaniappan, K. Stokes, M. Brandon and K. M. Ryan, ACS Nano, 2015, 9, 7456-7465. 30 D. Duveau, B. Fraisse, F. Cunin and L. Monconduit, Chem. Mater., 2015, 27, 3226-3233.

$31 \mathrm{M}$. Ge, S. Kim, A. Nie, R. Shahbazian-Yassar, M. Mecklenburg, Y. Lu, X. Fang, C. Shen, J. Rong, S. Y. Park, D. S. Kim, J. Y. Kim and C. Zhou, Nanotechnology, 2015, 26, 1-9.

32 T. Song, H. Cheng, T. Kaitlin, P. Hyunjung, R. W. Black, L. Sangkyu, W. Park II, Y. Huang, J. A. Rogers, L. F. Nazar and U. Paik, Adv. Funct. Mater., 2014, 24, 1458-1464.

33 T. Song, H. Cheng, H. Choi, J. H. Lee, H. Han, D. H. Lee, D. S. Yoo, M. Kwon, J. Choi, S. G. Doo, H. Chang, J. Xiao, Y. Huang, W. Park II, Y. Chung, H. Kim, J. A. Rogers and U. Paik, ACS Nano, 2013, 6, 303-309.

34 M. Haro, T. Song, A. Guerrero, L. Bertoluzzi, J. Bisquert, U. Paik and G. Garcia-Belmonteal, Phys. Chem. Chem. Phys., 2014, 16, 17930-17935.

35 W. Xiao, J. Zhou, L. Yu, D. Wang and X. W. Lou, Angew. Chem., 2016, 55, 7427-7431.

36 C. Xiao, N. Du, X. Shi, H. Zhang and D. Yang, J. Mater. Chem. A, 2014, 2, 20494-20499.

37 Y. Li, K. Yan, H. W. Lee, Z. Lu, N. Liu and Y. Cui, Nat. Energy, 2016, 1, 15029.

38 Y. Liu, B. Chen, F. Cao, H. L. W. Chan, X. Zhao and J. Yuan, J. Mater. Chem., 2011, 21, 17083-17086.

39 R. Xu, S. Wu, Y. Du and Z. Zhang, Chem. Eng. J., 2016, 296, 349-355. 